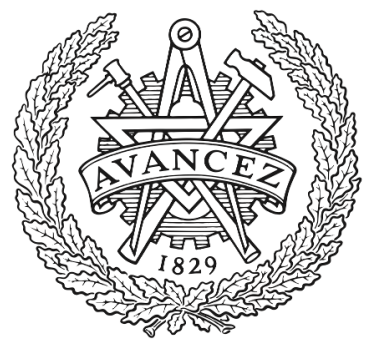

CHALMERS

UNIVERSITY OF TECHNOLOGY

\title{
Influence of body stiffness on vehicle dynamics characteristics
}

Downloaded from: https://research.chalmers.se, 2023-04-26 08:54 UTC

Citation for the original published paper (version of record):

Danielsson, O., González Cocaña, A., Ekström, K. et al (2016). Influence of body stiffness on vehicle dynamics characteristics. Dynamics of vehicles on roads and tracks. 24th Symposium of the International-Association-for-Vehicle-System-Dynamics (IAVSD), 2015. Graz; Austria, 17-21 August 2015: 45-56. http://dx.doi.org/10.1201/b21185-7

N.B. When citing this work, cite the original published paper. 


\title{
Influence of Body Stiffness on Vehicle Dynamics Characteristics
}

\author{
O. Danielsson \& A. González Cocaña \\ Vehicle Engineering \& Autonomous Systems, Chalmers University of Technology, Gothenburg, Sweden
}

K. Ekström, M. Bayani Khaknejad \& M. Klomp ${ }^{1}$

Vehicle Dynamics \& Active Safety Centre, Volvo Car Group, Gothenburg, Sweden

R. Dekker

Aerospace Structures \& Materials, Delft University of Technology, Delft, the Netherlands

\begin{abstract}
Body stiffness is one of is one of the characteristics of a passenger car affecting the handling, steering and ride comfort characteristics of the vehicle, but so far only limited aspects of mainly steering and handling has been analyzed. In this paper an extensive and detailed analysis of the influence of body stiffness on steering, handling and ride comfort is performed using a combination of laboratory and track testing of a physical prototype as well as sub-system and full vehicle multi-body simulation and finite element analysis. In this study it is found that the on-center, frequency response and primary ride are mostly affected by changes in body stiffness. For example, the torsional stiffness should be increased in order to improve roll balance in ride maneuvers. It is also found that the front and rear lateral stiffness should be raised in order to reduce dead band and steering wheel angle to yaw rate phase lags. Furthermore, maneuvers that combine handling and ride effects are proven to be particularly useful for defining body stiffness targets related to vehicle dynamics effects and one such maneuver is developed and evaluated in this paper. Finally, it is found that the difference between a rigid and flexibly vehicle model can be up to $13 \%$ for certain metrics, meaning that assuming a rigid body can be a significant oversimplification.
\end{abstract}

\section{INTRODUCTION}

Vehicle dynamics characteristics are essential for driving experience of the customer and may be subdivided into handling, steering and ride comfort characteristics. A major sub-system in vehicle is body-in-white and, in order to support the requirement setting of this sub-system, it is essential for both road [1-3] and rail vehicles [4-5] to properly understand the influence of local and global stiffness properties on vehicle dynamics characteristics.

From subjective physical testing it is known that the local and global body stiffness properties are important for vehicle dynamics characteristics. In [1] for instance, it was established that the increase in lateral and torsional stiffness both resulted in a significant improvement in subjective evaluation of overall dynamic vehicle response. This was in [1] and [2] further established with objective data where significant improvements in yaw gain and decrease in time lag was observed. Up to $14 \%$ of improvement in the phase lags of the vehicle for steering input frequencies of 0.5 $\mathrm{Hz}$ is seen through increasing the global torsional stiffness of the car. Regarding local stiffness, it is found that increasing the local stiffness at the front lower control arm attachment points also results in an improvement in overall vehicle response [2]. However during virtual objective testing of a different vehicle no significant change in yaw rate response (gain and response time) could be found [3] between a rigid body and that with a flexible body with a $50 \%$ reduction in the Young's modulus of the vehicle body.

Previous studies performed by Volvo Cars have shown an influence from body stiffness on subjective measurements taken during physical tests where the car was described as more solid and premium when reinforced.

According to Milliken [7], excessive chassis flexibility induces problems in the field of lateral load transfer, suspension kinematics and it results in unwanted vibrations. For a race vehicle,

\footnotetext{
${ }^{1}$ Corresponding author, e-mail: matthijs.klomp@ volvocars.com
} 
Milliken states that the chassis torsional stiffness can be approximately designed to be 3-5 times the total suspension roll stiffness. This cannot be directly transposed to a passenger car but it gives an approximation of the influence of the body stiffness on vehicle dynamics characteristics. Typical values for torsional stiffness in a passenger car are in the range of 17 to $40 \mathrm{kNm} / \mathrm{deg}$, with a roll stiffness of 1.0-2.5 kNm/deg per axle; resulting in a chassis/roll stiffness ratio of 6.8-40 [8].

Nevertheless, in the references above only a limited number of vehicle dynamics characteristic properties are considered; mainly related to the yaw response of the vehicle. Although important, the aim of this paper is to extend the study to properties of the vehicle on three different levels: component (body stiffness properties), sub-system level (such as suspension kinematics \& compliance) and linear full-vehicle analysis as well as full-vehicle ride $\&$ handling driving maneuvers (quasi-steady-state and dynamic) and to show how these are interrelated.

In the paper a systematic approach via an automated sensitivity analysis is employed in order to analyze and report the influence of local and global stiffness properties of the car body on suspension kinematics and compliance as well as full-vehicle ride and handling. The maneuvers that are studied consist of standard quasi-static and dynamic full-vehicle events for ride, steering $\&$ handling, suspension kinematics \& compliance analyses and four-poster vertical dynamics simulations. This is done using a multi-body model of the reference vehicle through an automated process such that the influence from body stiffness on ride, steering and handling can be assessed in a systematic way.

\section{METHOD}

From the introduction it is clear that understanding of the aforementioned properties still requires further work. Since, the aim of this paper is to analyze and understand the influence of local and global stiffness properties of the car body on suspension kinematics and compliance as well as full-vehicle ride, steering and handling characteristics, simplified analytical models (inspired by methods employed in [5]), high-fidelity multi-body simulation models and physical vehicle testing are employed to perform the study. The multi-body analysis is supported by modal reduction of a high-fidelity finite-element model of the vehicle body via the Craig-Bampton method [6]. This will be discussed further in section 2.2. In order to correlate the objective findings to subjective results, many of these changes are replicated in a physical vehicle of which objective measurements and subjective evaluations are carried out in parallel.

A repeatable way of evaluating vehicle dynamics is needed in order to be able to identify the influence of small design changes. Hence, multiple standard maneuvers and metrics are used. In this study vehicle behavior is divided in three different attribute groups, where the corresponding performance is measured by executing different maneuvers. These attribute groups are handling, steering and ride. For each of these three groups a set of maneuvers are performed in order to evaluate different attributes. From these maneuvers a set of scalar metrics are calculated for each attribute with the help of a post processing tool. The calculated metrics are then used to assess vehicle performance in handling, steering and ride.

The software used for the graphical visualization and correlation of large data sets of results from the performed design of experiments (DoE) is ModeFrontier. This multi-objective optimization software contains a variety of tools used to perform statistical analysis. These tools are used to explore the design space and to analyze results. Data and data distributions can be visualized using scatter, bubble plots, histograms, cumulative plots and student charts.

The vehicle used in this study is a Volvo S60 as shown in Figure 1. A physical vehicle was modified with detachable steel bars allowing to increase or decrease the body stiffness in specific areas. Meaning that the same vehicle can be compared against a baseline but with an increment or decrement in body stiffness. These stiffeners are divided into six groups as shown in Table 1. 


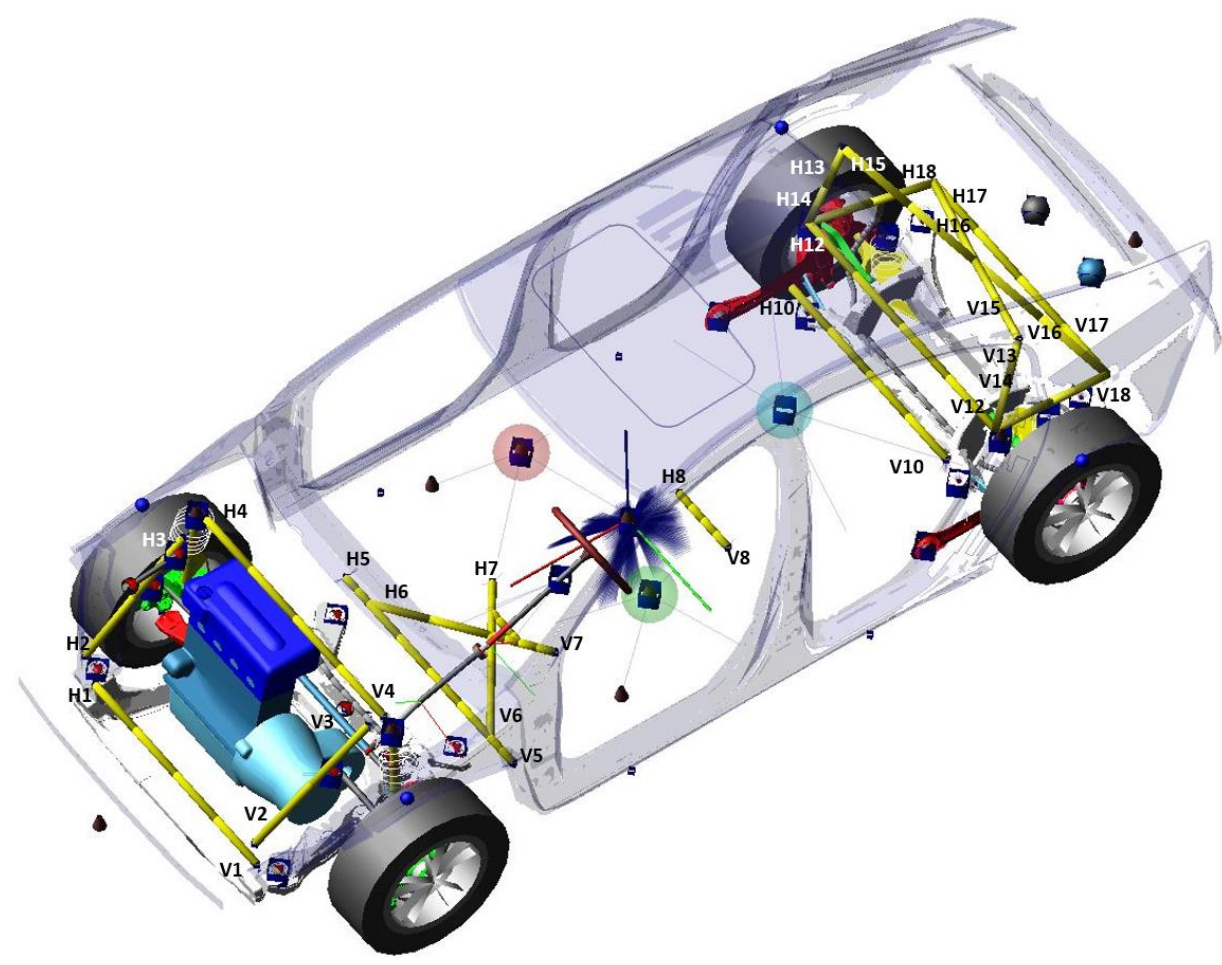

Figure 1 - Vehicle model with reinforcements used in paper

Table 1 - Body stiffener groups

\begin{tabular}{|l|l|l|}
\hline Group & Reinforcements & Numbering \\
\hline Front Longitudinal & 2 longitudinal bars & H2-H3, V2-V3 \\
\hline Front Transversal & 2 transversal bars & H1-V1, H4-V4 \\
\hline Underbody & 1 cross section \& 2 longitudinal bars & H6-V7, V6-H7, H5-V5, H8-V8 \\
\hline Rear Longitudinal & 2 longitudinal bars & V14-V18, H14-H18 \\
\hline Rear Transversal & 3 transversal bars & V10-H10, V12-H12, V17-H17 \\
\hline Rear Cross & 1 cross section & $\begin{array}{l}\text { V16-H15, H16-V15, V13-V14, } \\
\text { H13-H14 }\end{array}$ \\
\hline
\end{tabular}

The CAE analysis method used for correlating body stiffness and vehicle dynamics characteristics is divided in three different relations levels: component to sub-system, sub-system to vehicle and component to vehicle. The component level describes the local stiffness of the body i.e. how the different stiffeners increase body local stiffness; the sub-system level corresponds to global stiffness and $\mathrm{K} \& \mathrm{C}$ values i.e. lateral compliance, toe compliance, camber compliance, bending stiffness or torsional stiffness; and the vehicle level assesses the dynamic behavior of the vehicle in terms of handling, steering and ride.

Accordingly, detailed analyses of the effect of the reinforcements on the torsional and bending stiffness, kinematics \& compliance as well as steering, handling and ride characteristics are performed. This analysis has been subdivided such that the full-vehicle analysis was performed with a vehicle model (IPG CarMaker) that uses sub-system characteristics as input values. Since carmaker benefits a form of lookup table representation of the suspension system, this provides computationally efficient model for sensitivity analysis, And hence a full factorial analysis of six groups of reinforcements (shown in Table 1) and 14 vehicle maneuvers (with selected metrics of each maneuver being the output) could be analyzed. The sub-system characteristics that are inputs to the simulation model are the body bending and torsional compliance (obtained from a finiteelement analysis of the body-in-grey) and the translational and rotational compliance of the wheel center (obtained from a multi-body simulation model). These different sources of data to the model used for full-vehicle steering, handling and ride analysis are illustrated in Figure 2. 


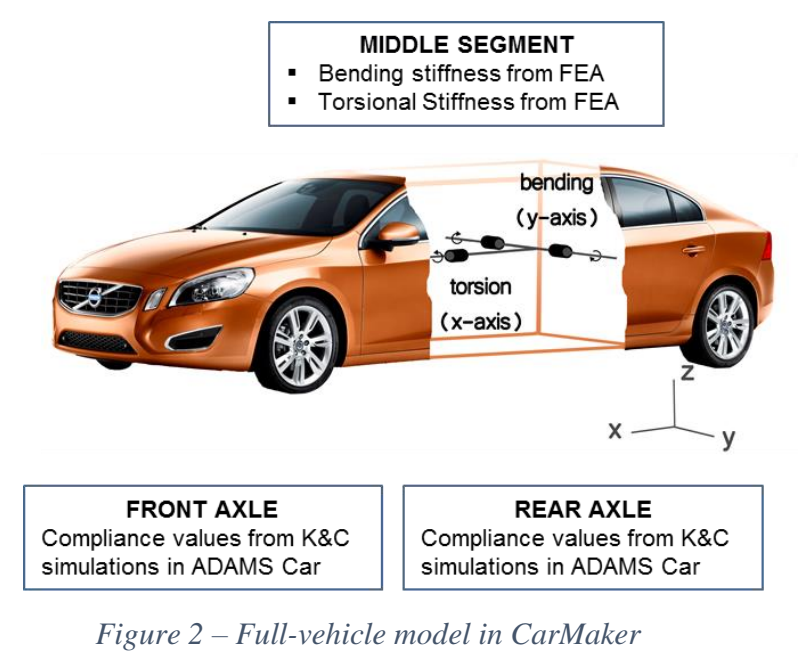

\subsection{Torsional and Bending Stiffness Computation using Finite Element Analysis}

In order to obtain the bending and torsional stiffness of the vehicle body, a detailed finite element model was used. The finite element method (FEM) model used for calculating the static stiffness has approximately 1 million elements and it represents the body-in-grey (BIG) of the Volvo S60. It has been reinforced in rig mounting areas in order to avoid local deformation while performing the static stiffness tests. Specifically, the top mounts and the force application area in the floor for the three point bending test have been reinforced. The reinforcement has been done by adding rigid body elements using RBE2 constraint in mentioned areas (rigid body with independent DOF at one GRID and dependent DOF in the slave nodes around). The stiffeners which decrease the body compliance are modeled using CBAR elements, simple beam connections. They connect the different points in the body-in-white in the same way as for the physical test vehicle.

Finite element analysis is performed using MSC Nastran solver in order to calculate global torsional and bending body stiffness. In fact, these two parameters are not completely captured in kinematics \& compliance $(\mathrm{K} \& \mathrm{C})$ measurements, since the body is clamped in the middle section when $\mathrm{K} \& \mathrm{C}$ tests are performed. The borders between where kinematics \& compliance and respectively the FEA simulations will account for body stiffness are somewhat diffuse, for example the roof structure will not be clamped during the kinematics \& compliance tests while the underbody in the middle area is in fact clamped. For this reason the values for torsional and bending stiffness are going to be considered as global body properties. The torsional stiffness test and the three point bending test shown in Figure 4 are performed in CAE in order to calculate values for these two static properties. Furthermore only static stiffness tests are performed due to that handling, steering \& primary ride events operates at a frequency below $20 \mathrm{~Hz}$; and the first body natural frequency is located around $40 \mathrm{~Hz}$.
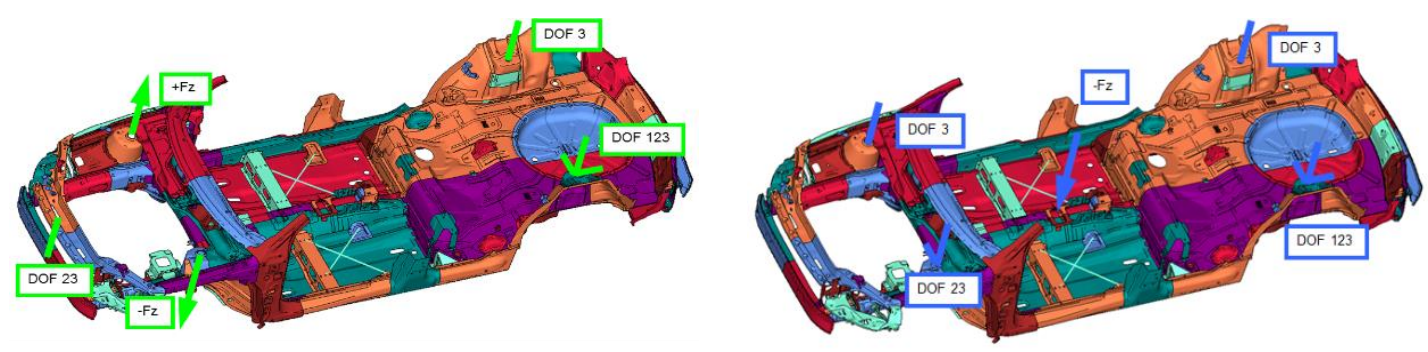

Figure 3 - Torsional stiffness test (left) and three-point bending test (right) 


\subsection{Front and Rear Suspension Kinematics \& Compliance Analysis}

The kinematics \& compliance $(\mathrm{K} \& \mathrm{C})$ analysis is done using a multi-body simulation (MBS) model developed in Adams/Car. The main sub-systems in the vehicle model are the front and rear suspensions, steering mechanism and the vehicle body. The front suspension system in the Volvo S60 is a McPherson strut suspension. All suspension linkages and the sub-frame are modelled as super element flexible bodies including up to 34 and 112 modes, respectively. The rear suspension is a multi-link suspension where all linkages, including knuckle assembly, spring link, toe link and camber link are similarly modelled as super element flexible bodies with modal information for 30 modes. Rear sub-frame and trailing arm has modal information up to 60 modes. The vehicle model is shown in Figure 1.

As described in the introduction, the multi-body model uses a modal reduction representation of the body from an FEM model in order to simulate these effects. The super element representation of the body-in-grey corresponds to the baseline body-in-grey of a Volvo S60. In this case the modal information for the S60 baseline body is containing 221 modes.

In order to study the mechanical behavior of vehicle systems, substituting rigid components for their flexible representatives would provide higher degrees of freedom (DOF) in the model. Taking advantage of this opportunity, modal and mechanical responses of components can be calculated in large multi-body motion simulations. However, this in turn causes higher calculation cost and time.

The main idea in FE reduction method is to choose a series of interface nodes and rewrite the stiffness matrix in a reduced form. The boundary nodes (or interface nodes) can be a combination of attachment points or specific nodes of high interest for monitoring.

In the MBS model this reduction is done by using MNF models (Modal Natural File). The important assumption is that local deformations can be approximated as a linear combination of limited number of shape vectors. For this purpose, Craig-Bampton method is implemented, in which the stiffness matrix is partitioned to two sections of boundary DOF and interior DOF. And the shape vectors are defined in the form of two different sets:

Constraint modes: that are the shape vectors from static deformation of the part obtained by applying a unit displacement at each boundary DOF while all other boundary DOFs are fixed.

Fixed-boundary eigen modes: that are obtained by a simple eigen solution while all boundary DOFs are fixed. Needless to say that the quality of this modal expansion is proportional to the number of eigen modes retained in the model for reduction purpose.

For the reinforced car model, the study has been performed by reinforcing the body-in-white with different bars, in the similar manner as in the physical test car. The tube thickness of the bars is $2 \mathrm{~mm}$ with an external diameter of $15 \mathrm{~mm}$. However, these reinforcements are modeled in Adams/Car with rigid beams i.e. rigid connections connecting the two desired points of the bodyin-white. Selecting rigid beams in Adams/Car was due to the fact that it provides a better correlation between the compliance values obtained in the SPMM rig and CAE simulations performed in Adams/Car.

Two vertical wheel travel tests (parallel \& single) and five compliance tests (longitudinal and lateral translation as well as camber, toe and spin rotation) were performed and the compliance in the design position of each suspension (front and rear) were used as inputs values for the fullvehicle simulation in CarMaker. 


\subsection{Handling, Steering \& Ride Analysis in CarMaker}

To objectively evaluate the vehicle dynamics characteristics of the vehicle, three categories are used: handling, steering and ride. The used maneuvers are: Braking in a Turn (ISO 7975:2006), Ramp Steer and Constant Radius (ISO 4138:2012 and ISO/DIS 19364), Constant Radius with Bump, Frequency Response (ISO 7401:2011), Sine-with-Dwell (ISO/DIS 19365), On-center Handling (ISO 13674-1:2010 and ISO 13674-2:2006), Throttle Release in Turn (ISO 9816:2006) and Primary Ride (ISO 2631-4:2001). The maneuvers are implemented in CarMaker and evaluated to retrieve the metrics for each maneuver.

Of the above tests, the only non-standard maneuver is the constant radius with speed-bump. Constant radius with bump is performed similar to "Constant radius" but at constant speed and with a bump positioned transversally on the track. The transient condition occurring when hitting the bump symmetrically (left/right wheels simultaneously) and asymmetrically (left/right wheels sequentially) is analyzed. More details on this maneuver can be found in [10].

\section{RESULTS}

One challenge when the DoE contains a large number of inputs and outputs is to summarize information about data in an effective way. Common statistical functions i.e. mean, confidence, interval, the quartiles, outliers and the effect are used for this purpose.

In this section subjective and objective test results are presented. Also, the correlation with physical measurements is presented.

\subsection{Subjective Testing}

Subjective testing is performed on the Hällered proving ground in order to obtain a subjective evaluation of the vehicle dynamics behavior of the car with different configurations of reinforcements. The subjective testing is carried out by professional test drivers and the comments are analyzed together with subjective ratings. It is intended to match the subjective results with the objective measurements and CAE analysis. Some example comments for the subjective analysis for vehicles when increasing the body stiffness are:

- The car feels more solid

- Premium / quality feeling

- Less harshness feeling

- More compact and agile

- More connected

- Steering compliance is much reduced

The comments indicate in general that the vehicle performance is increasing when increasing the body stiffness. They suggest a more controlled and responsive car. There are, however, some changes require the vehicle to be retuned for the new condition. For example, the cross-bars in the rear compartment improve shake but also reduce understeer and thereby reduce the stability and increases the phase-lags. Also, some of the changes to the front part of the vehicle make the steering response too progressive and re-tuning is required to come back to a linear response.

As stated in the introduction, the question now is this subjective feeling can be correlated to changes in objective vehicle dynamics metrics, which is the topic for the following sections. 


\subsection{Compliance and global torsional and bending stiffness}

This section presents results from both the MBS model and the FEM model and it contains results connecting component characteristics to subsystem characteristics. The MBS model is used to capture the effect on lateral and local stiffness from adding stiffening bars to the BIG, the resulting groups seen in Figure 4 are: Lateral Stiffness - Front, Lateral Stiffness - Rear, Camber Stiffness - Front, Camber Stiffness - Rear, Toe Stiffness - Front and Toe Stiffness - Rear. In order to also capture the relation between stiffening bars and global torsional and bending stiffness values, results from the FEM model are also included in Figure 4; these results are represented by two groups: Global Torsion Stiffness and Global Bending Stiffness. The groups connected to lateral and local stiffness were chosen based on the effect of the bars on each compliance set of parameters.

The results in Figure 4 show how each stiffener affects compliance values. It can be seen that Front Transversal, Rear Cross, Underbody and Rear Longitudinal are the main groups of bars affecting the stiffness of the BIG. Global stiffness values are affected by the Rear Cross, Underbody and Rear Longitudinal bars. The bars located under the middle part of the car together with the Rear Longitudinal bars largely affect global bending stiffness whilst the Rear Cross is affecting global torsional stiffness. Regarding lateral and local stiffness values: they change to a lesser extent than the global stiffness values, with Front Transversal showing the largest effect on the lateral and local stiffness parameters. These results also show that bars from the groups: Front Longitudinal, Rear Longitudinal and Rear Transversal barely has any effect on local or global compliance values.

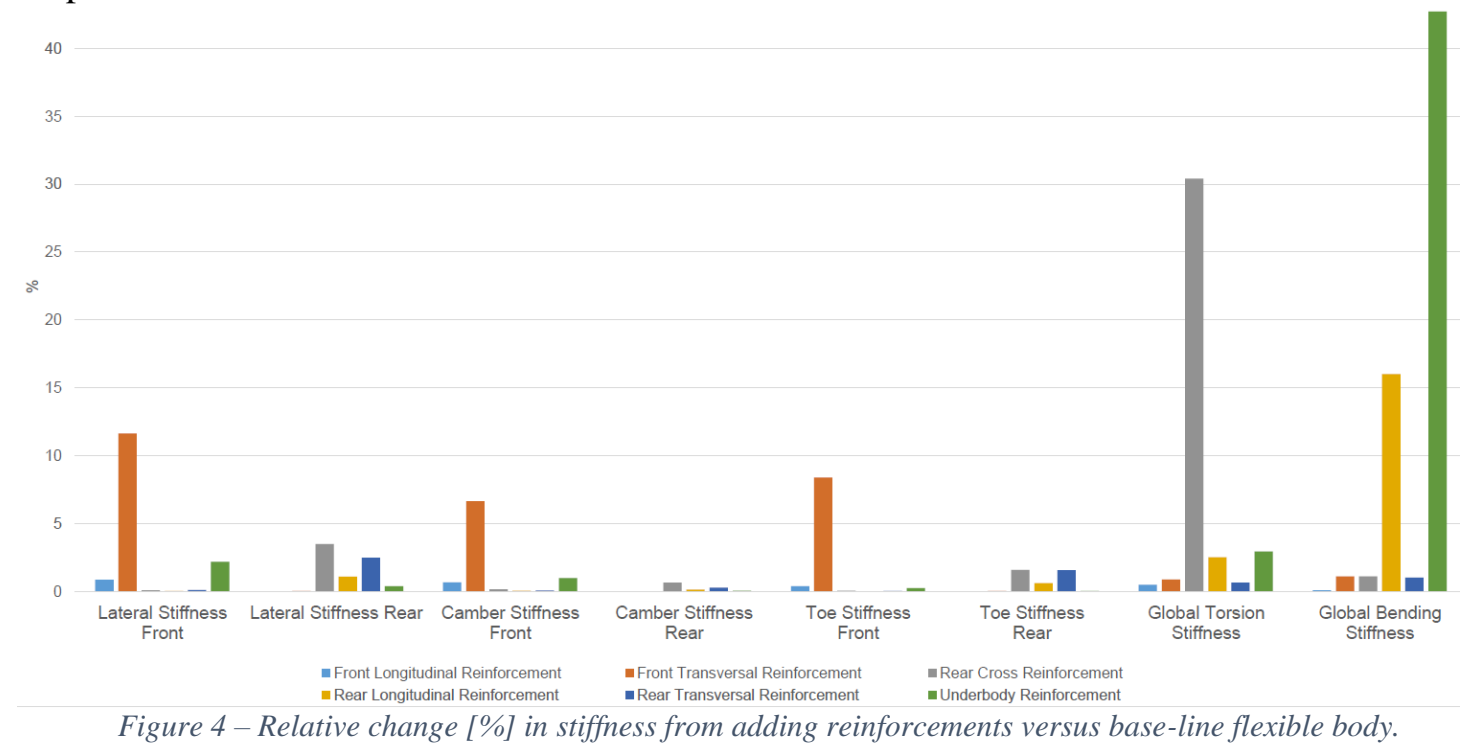

Figure 6 shows the percentage difference in stiffness between the baseline Volvo S60 vehicle and the same vehicle fitted with all body reinforcements, for both physical tests and CAE simulations. As can be seen in Figure 6 both the compliance and the torsional stiffness values from the CAE simulations correlate well with the results obtained from the physical tests. 


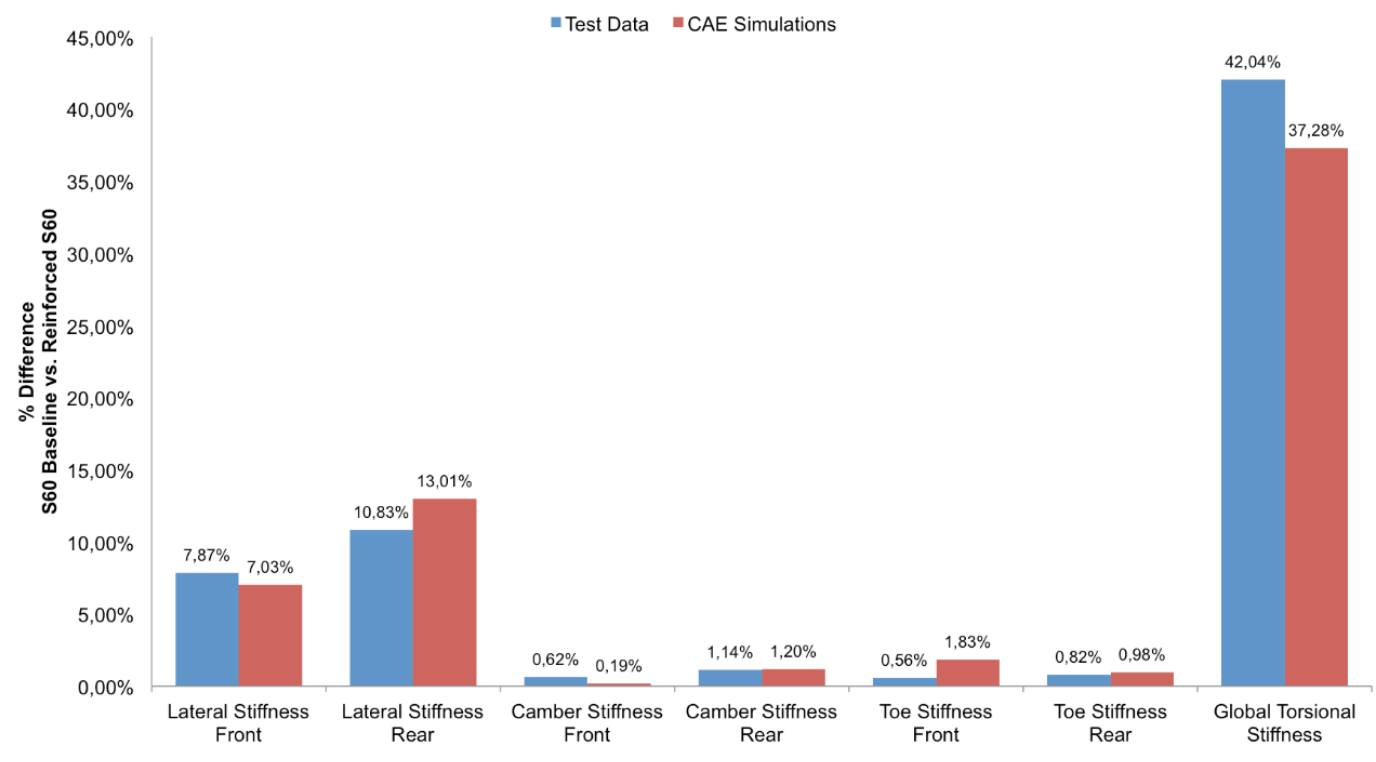

Figure 5 - Stiffness correlation, measurement versus simulation.

\subsection{Handling, steering and ride analysis}

This section presents the results from the CarMaker model simulations described in the Method section. It relates subsystem characteristics to vehicle dynamics characteristics. This relation can be translated into the relation between $\mathrm{K} \& \mathrm{C}$ and global stiffness with handling, ride and steering characteristics. In order to make this connection, simulation groups containing compliance values related to load cases were created. In other words, these results present the relation between the local stiffness, lateral stiffness, torsional stiffness and bending stiffness of the BIG; and the vehicle dynamics behavior of the vehicle.

CarMaker simulations are conducted with a fully rigid body, reinforced body and with the baseline Volvo S60 body. The reinforced body configuration and the rigid body are compared with the baseline $\mathrm{S} 60$ body.

Figure 6 show results from the six metrics with the largest percentage change when adding/removing stiffeners and for making the body completely rigid. The values are normalized against desired target range to take into account the sensitivity of the overall car behavior to each metric. These six metrics are connected to the following maneuvers: primary ride, on-center and constant radius. On center yaw gain, Steering sensitivity, Off center yaw gain and Deadband in degrees are mostly affected by Lateral Front, Lateral Rear and Camber Front. The largest factors for Roll balance from primary ride is global torsional and bending stiffness whilst the understeer ratio from the constant radius test is mostly effected by Bending, Lateral Front, Lateral Rear and Camber Front. 


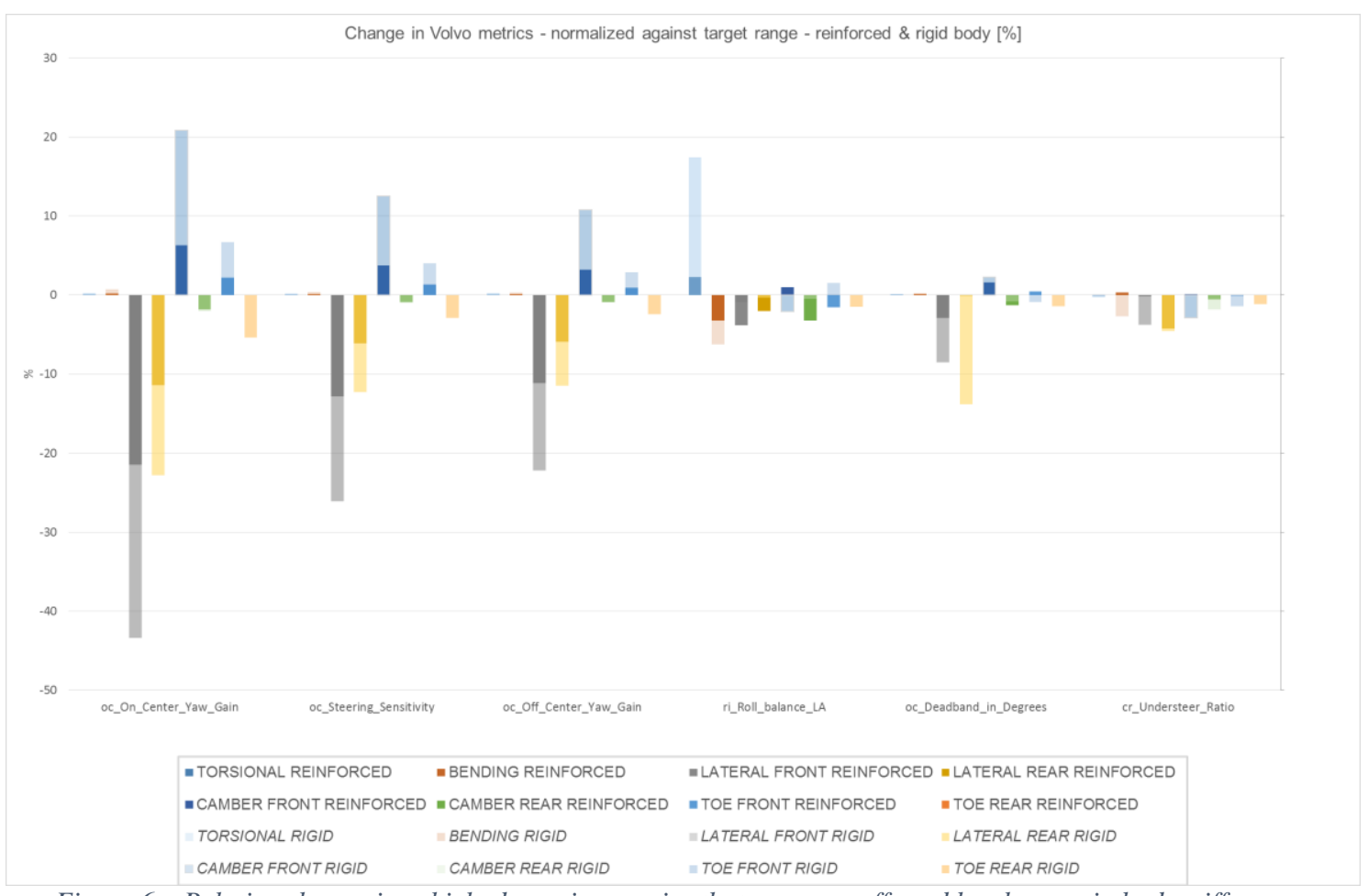

Figure 6 - Relative change in vehicle dynamics metrics that are most affected by changes in body stiffness - normalized against target range - reinforced \& rigid body [\%]

As described in the methods section, it is hypothesized (based on analysis of subjective evaluation) that maneuvers combining ride comfort and handling maneuvers would be more influence by changes in the body stiffness than many of the standard maneuvers. The results in Figure 7 shows that the largest metric percentage change in new metrics is calculated in maneuvers: Constant radius with bump and constant radius with angled bump. The most influencing factors are the global values for torsional and bending stiffness.

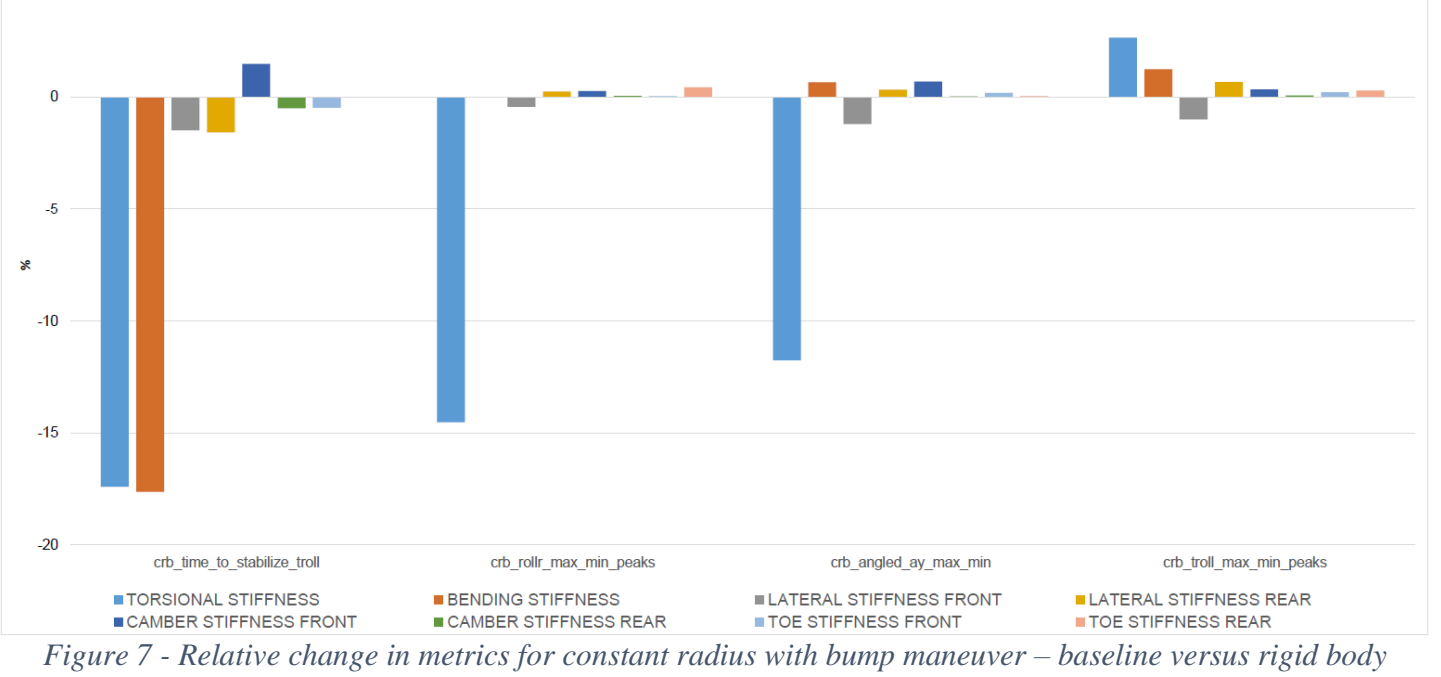

To ensure that the vehicle model is implemented correctly and that it is producing reasonable and accurate results it was verified and validated with experimental data as well. In order to verify the model, output metric values were checked for reasonable results for various input parameters in different driving maneuvers. The model was also validated with data from physical tests to ensure the accuracy of the model. This was done by performing a set of standard maneuvers with the simulation model and compare the results to data gathered from objective physical tests. 
Results from the validation maneuvers are shown in Figure 8, where it can be seen that there is good agreement in the relative change between simulation and measurement. It can further be seen that, in all cases except for the steering angle dead-band, the simulation under-predicts the change in metrics. In particular this appears to be the case for understeer of the vehicle, which is known to be sensitive to small changes in the front to rear compliance.

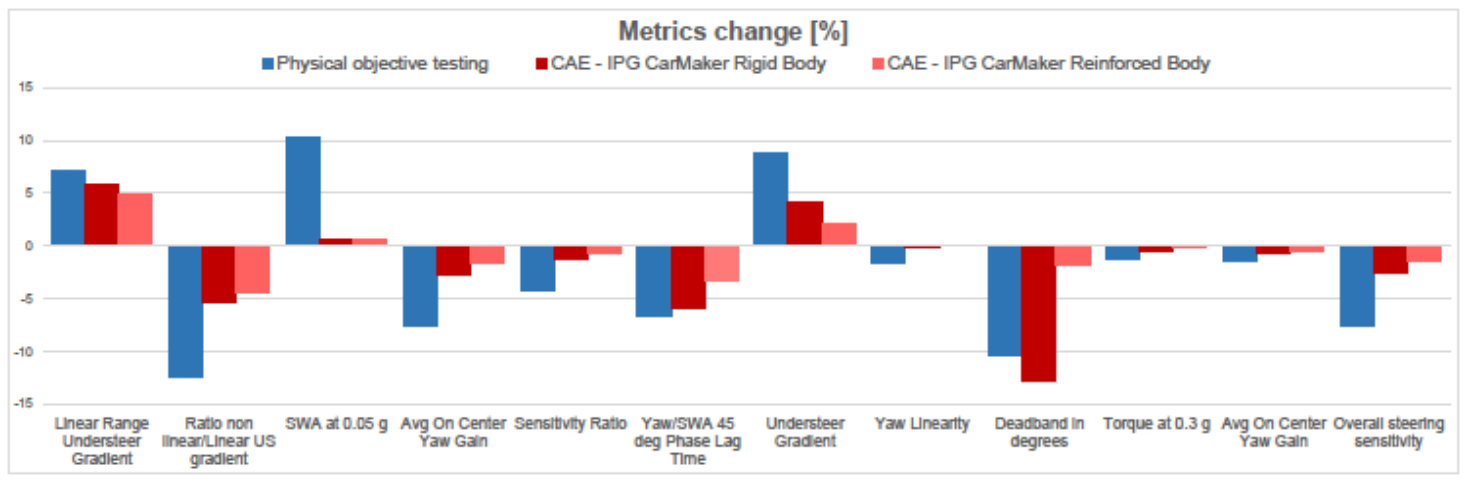

Figure 8-Relative change in metrics [\%] of physical vehicle testing versus simulation (CAE)

\section{SUMMARY AND CONCLUSIONS}

In summary, the main conclusions from this study are that:

- Full vehicle CAE simulations performed with the simulation tool IPG CarMaker show a significant difference in vehicle dynamic characteristics when comparing a passenger car with a reinforced body against a baseline Volvo $\mathrm{S} 60$ vehicle.

- On-center and primary ride are one of the most affected maneuvers while evaluating body stiffness vs. vehicle dynamics.

- Global torsional stiffness should be increased in order to improve roll balance in ride maneuvers.

- Front and rear lateral stiffness should be raised in order to reduce Deadband and Steering Wheel Angle - Yaw phase lags.

- Physical measurements from objective testing follow the same trends as seen in CAE simulations in IPG CarMaker for full vehicle maneuvers including handling, steering and primary ride effects.

- Body stiffness can be captured to an acceptable level for handling, steering and ride maneuvers by using a model where global torsional and bending stiffness are taken into account by a joint between two rigid bodies and local stiffness effects are taken into account in the compliance values of the suspension. However a more detailed body model based on FEM and modal reduction is needed for a better correlation in absolute numbers and not only in trends when performing dynamic simulations.

- Maneuvers combining handling and ride effects are proven to be useful for defining body stiffness targets according to vehicle dynamics effects.

- There is a significant error factor in full vehicle dynamics simulations when the vehicle body is assumed to be rigid. This error can be estimated to up to $13 \%$ for certain standard metrics. The accuracy of the simulation results can be increased by using a flexible body instead of a rigid body for full vehicle simulations.

- Global torsional stiffness is not the only main parameter when defining body stiffness targets and requirements for a better vehicle dynamics behavior. Lateral stiffness shows to have a large effect on the dynamic behavior and should be considered when defining body stiffness goals.

- Reinforcements fitted to a vehicle body can be seen to affect different aspects of vehicle performance with altering magnitude and relation dependent on where they are 
fitted i.e. different bars can counteract each other's effect on vehicle dynamics characteristics. Resulting in that the vehicle can be tuned during development using body stiffness as a design variable.

- The CAE method used for this study based on first exploring the design space with a simple model and secondly perform a deeper analysis in the interesting areas with a more complex model can be translated to study other areas of the vehicle. Thus, this method can be useful to reduce the development time of a vehicle and to increase the understanding of design choices in a complex system.

\section{ACKNOWLEDGEMENTS}

The authors would like to thank Volvo Car Group for sponsoring this study and for the possibility to use the collected data to verify our results. The author's would also like to thank the contribution of the following individuals: Bengt Jacobson and Mathias Lidberg from Chalmers University and Johan Hultqvist, Stavros Angelis and Åsa Eriksson at Volvo for support and guidance in this project. Furthermore Niklas Johansson for supplying the finite element model and supporting torsional and bending stiffness analysis as well as Axel Jonson and Daniel Hedendahl at Volvo for their help with the post-processing of the vehicle dynamics simulation results.

\section{REFERENCES}

[1] Sreeraj, N., et al. Establishing Correlation between Torsional and Lateral Stiffness Parameters of BIW and Vehicle Handling Performance. No. 2011-01-0089. SAE Technical Paper, 2011.

[2] Chaturvedi, Bhaskar, Deepak Rana, and Mugundaram Ravindran. Correlation of vehicle dynamics \& NVH performance with body static \& dynamic stiffness through CAE and experimental analysis. No. 2010-01-1137. SAE Technical Paper, 2010.

[3] Coox, Laurens, et al. "Numerical assessment of the impact of vehicle body stiffness on handling performance." Proceedings of ISMA2012 (2012): 3711-3724.

[4] Persson, Ingemar, and Mats Holgersson. "Body structure and vehicle dynamics." Vehicle System Dynamics 15.sup1 (1986): 441-447.

[5] Yadav, D., S. Kamle, and S. Talukdar. "Heave-pitch-roll dynamics of flexible vehicle in variable velocity run." Vehicle System Dynamics 33.1 (2000): 1-28.

[6] Bampton, Mervyn CC, and Roy R. CRAIG, JR. "Coupling of substructures for dynamic analyses." AIAA Journal 6.7 (1968): 1313-1319

[7] Milliken, William F., and Douglas L. Milliken. Race Car Vehicle Dynamics. Vol. 400. Warrendale: Society of Automotive Engineers, 1995.

[8] Dixon, John C. "Tires, suspension and handling." Training 2015 (1996): 06-15.

[9] Benjamini, Yoav. "Opening the Box of a Boxplot." The American Statistician 42.4 (1988): 257-262.

[10] Danielsson, Oskar and González Cocaña, Alejandro. "Influence of Body Stiffness on Vehicle Dynamics Characteristics in Passenger Cars". Diploma work - Department of Applied Mechanics, Chalmers University of Technology, Göteborg, Sweden, ISSN 1652-8557; 2015:68, 2015. 